ARTICLE

https://doi.org/10.1038/s41467-019-12487-w

\title{
Directing isomerization reactions of cumulenes with electric fields
}

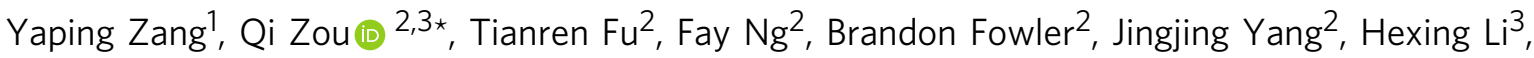 \\ Michael L. Steigerwald ${ }^{2 \star}$, Colin Nuckolls (1) ${ }^{2 \star} \&$ Latha Venkataraman (1) ${ }^{1,2 \star}$
}

Electric fields have been proposed as having a distinct ability to catalyze chemical reactions through the stabilization of polar or ionic intermediate transition states. Although fieldassisted catalysis is being researched, the ability to catalyze reactions in solution using electric fields remains elusive and the understanding of mechanisms of such catalysis is sparse. Here we show that an electric field can catalyze the cis-to-trans isomerization of [3] cumulene derivatives in solution, in a scanning tunneling microscope. We further show that the external electric field can alter the thermodynamics inhibiting the trans-to-cis reverse reaction, endowing the selectivity toward trans isomer. Using density functional theory-based calculations, we find that the applied electric field promotes a zwitterionic resonance form, which ensures a lower energy transition state for the isomerization reaction. The field also stabilizes the trans form, relative to the cis, dictating the cis/trans thermodynamics, driving the equilibrium product exclusively toward the trans.

\footnotetext{
${ }^{1}$ Department of Applied Physics, Columbia University, New York, New York, USA. ${ }^{2}$ Department of Chemistry, Columbia University, New York, New York, USA. ${ }^{3}$ Shanghai Key Laboratory of Materials Protection and Advanced Materials in Electric Power, Shanghai University of Electric Power, Shanghai 200090, China. *email: qzou@shiep.edu.cn; mls2064@columbia.edu; cn37@columbia.edu; Iv2117@columbia.edu
} 
nderstanding and controlling electronic reorganizations in chemical reactions is at the heart of synthesis chemistry ${ }^{1}$. In principle, electric fields can catalyze chemical transformations through stabilizing otherwise unfavorable electronic structures, enabling new transition states, modifying reaction barriers ${ }^{2-5}$, and altering reaction selectivity ${ }^{5-9}$. For example, studies in electrochemical environments suggest that interfacial electric fields can be exploited to control the selectivity of non-faradaic reactions ${ }^{10,11}$, whereas vibrational Stark effect spectroscopy ${ }^{4}$ and single-crystal diffraction experiments ${ }^{12}$ have been utilized to measure the magnitude of the electric field at enzyme active sites and cocrystal systems. Although it is conceptually appealing and, in principle, widely applicable, the use of oriented electric fields to alter the electronic structure of molecular reactants and transition states is beset with challenges ${ }^{13,14}$. In this regards, the scanning tunneling microscopy (STM) offers an ideal platform with which to apply a large external electric field over the small distance between two electrodes to induce chemical transformations that can be probed directly by measuring singlemolecule junction conductance. Although a previous STM-based study has demonstrated a field-induced single-molecule reaction, it was limited in scope due to an ultra-low conversion rate (a few molecules per hour $)^{2,15}$.

The study based on STM-break junction (STM-BJ) technique described herein demonstrates that electric fields can direct and catalyze solution-phase reactions. We focus on [3]cumulene derivatives, which consists of chains of carbon atoms bound together to give contiguous $\pi$-bonds ${ }^{16}$, and follow the cis-to-trans isomerization reactions under an electric field. The experiments are performed using the STM-BJ technique ${ }^{17,18}$, which possesses the advantage of being able to apply a large (and possibly catalytic) electric field, while at the same time being able to detect (through measurements of single-molecular junction conductance) the chemical products of its catalysis. We also analyze the reactants and products formed within the STM setup using high-performance liquid chromatography (HPLC) and confirmed that the reaction indeed occurs in the solution phase. We show that, at room temperature, an electric field accelerates the otherwise extremely sluggish cis-to-trans isomerization of the cumulene. Density functional theory (DFT)-based calculations confirm that the electric field alters the [3]cumulene charge distribution promoting an ionic resonance form, thereby decreasing the isomerization barrier. The applied field also alters the reaction thermochemistry, favoring the trans form and determining the ultimate distribution of the products to one that cannot be achieved by simply heating or irradiating the solution.

\section{Results}

Synthesis of cumulenes. We design and synthesize an asymmetric [3]cumulene that has both an aurophilic (SMe) and an alkyl ( $\mathrm{t}-\mathrm{Bu}$ ) substituent on each end. Each SMe terminal group binds to undercoordinated Au atoms on the STM tip and substrate, enabling single-molecule junction conductance measurements, whereas the t-Bu group provides steric bulk to prevent unwanted reactions of the cumulated double bonds to ensure air stability $^{19}$. The synthesis, detailed in the Supplementary Methods, yields a mixture of the cis and trans isomers, cis[3] and trans[3]. We separated the isomers by fractional crystallization to isolate the pure cis[3] (23\% yield) and trans[3] forms (28\% yield), both of which are air stable as evidenced by $1 \mathrm{H}$-nuclear magnetic resonance (NMR) experiments (see Supplementary Methods).

Conductance measurements. STM-BJ measurements are made using a custom apparatus ${ }^{20}$ that applies a large electric field to the molecular solution in the region between the STM tip
(Fig. 1a) and substrate, and determines the conductance of single-molecule junctions (Fig. 1b) ${ }^{18}$. We describe the measurements, which are carried out at room temperature and in the dark, in the Methods section and Supplementary Note 1. In Fig. 1c, we present the single-molecule conductance data of cis [3] and trans[3] measured (separately) in tetradecane, a nonpolar solvent, at a tip bias of $0.1 \mathrm{~V}$. The illustrative conductance traces (Fig. 1c inset) start with plateaus close to integer multiples of $\mathrm{G}_{0}\left(=2 \mathrm{e}^{2} / \mathrm{h}\right.$, conductance quantum $)$ and show a molecular conductance plateau around $10^{-4} \mathrm{G}_{0}$. Logarithmically binned one-dimensional (1D) conductance histograms of such traces, compiled without any data selection from thousands of traces, collected within a $2 \mathrm{~h}$ period are shown in the main panel. The peak around $10^{-4} \mathrm{G}_{0}$ in the $1 \mathrm{D}$ histograms corresponds to the conductance of the single-molecule junctions. The peak for cis[3] is broad, whereas that for trans[3], at a slightly higher conductance, is more well-defined. We attribute the similar conductance value of the two isomers to their similar electronic structure, as can be deduced from their electrochemical and optical characterizations and their DFT-based frontier orbitals (see Supplementary Figs. 1-3).

The distinct conformations for cis[3] and trans[3], as determined by DFT-optimized molecular structures (see Methods) are shown in Fig. $1 \mathrm{~b}$ with model electrodes to illustrate the length of a junction. The S-S distance of cis[3] and trans[3] differs by about $0.6 \mathrm{~nm}$. This results in the conductance plateaus having different lengths as seen in the inset of Fig. $1 \mathrm{c}$ and in the two-dimensional (2D) conductance-displacement histograms (Fig. 1d, e). Length profiles of the 2D histogram features, shown in the inset of Fig. 1d, e, indicate that trans[3] junctions are roughly $0.6 \mathrm{~nm}$ longer than cis[3] ones. The experimental plateaus are shorter than the DFT-based junctions due to the gap that is formed after the Au-contact ruptures and relaxes ${ }^{20}$. Given the similar junction formation probability $(>94 \%$, see Supplementary Fig. 4 for the analysis), the longer junctions of trans[3] results in a stronger and more pronounced $1 \mathrm{D}$ histogram peak in contrast to that of the cis[3]. Taken together, these results illustrate the ability of the STM-BJ measurement to distinguish between cis[3] and trans[3] isomer-based molecular junctions.

Isomerization reactions of cumulenes. Focusing on the measurements of cis[3], we find that the conductance histograms change over time. Figure $2 \mathrm{a}$ and Fig. $2 \mathrm{~d}$ show $1 \mathrm{D}$ and $2 \mathrm{D}$ histograms compiled from 10,000 traces measured in $\sim 2 \mathrm{~h}$ at different times over the $32 \mathrm{~h}$ period. The histogram peak heights and the conductance plateau lengths increase with time. After about $32 \mathrm{~h}$, the conductance features are remarkably similar to those of trans[3] and do not change further. This change is also verified through conductance step length analysis of the 155,000 traces measured over the $32 \mathrm{~h}$ period (Fig. 2b). By contrast, a similar measurement of trans[3] lasting $60 \mathrm{~h}$ does not show any change (see Supplementary Figs. 5 and 6). We hypothesize that a selective cis-to-trans isomerization reaction takes place in our experimental setup.

To validate this hypothesis, we measure mixed solutions of cis [3] and trans[3] in which we vary their relative concentrations. The 1D and 2D histograms (generated by compiling thousands of traces collected in $<2 \mathrm{~h}$ for each solution) show conductance peak heights and plateau lengths increase as the concentration of the trans isomer is increased (Fig. $2 \mathrm{c}$ and Supplementary Fig. 7). This result compares well with that of the pure cis[3] solution measured as a function of time (Fig. 2a, d), supporting the isomerization reaction from cis-to-trans. Comparing these data and examining the plateau length distribution of the cis[3] and 
a

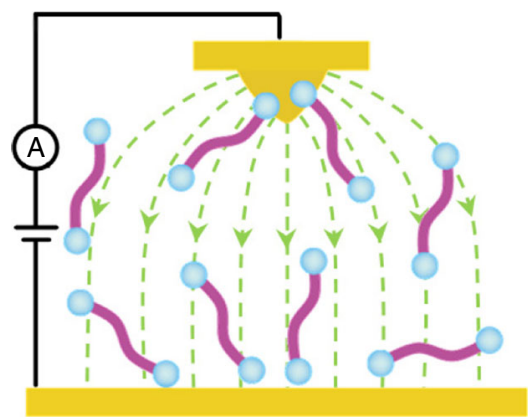

b

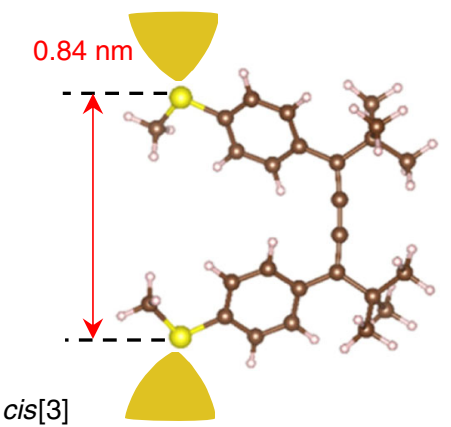

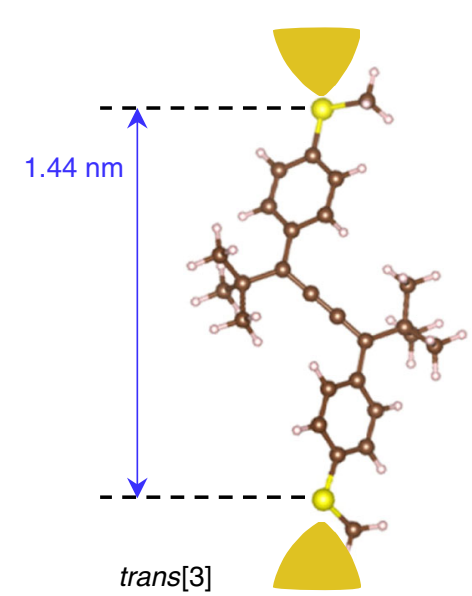

e

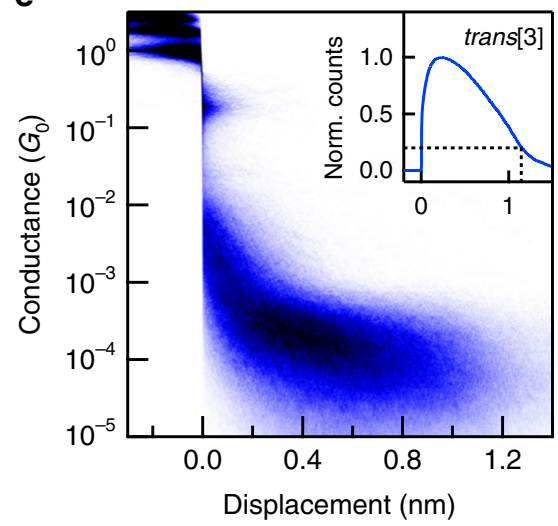

Displacement $(\mathrm{nm})$
C

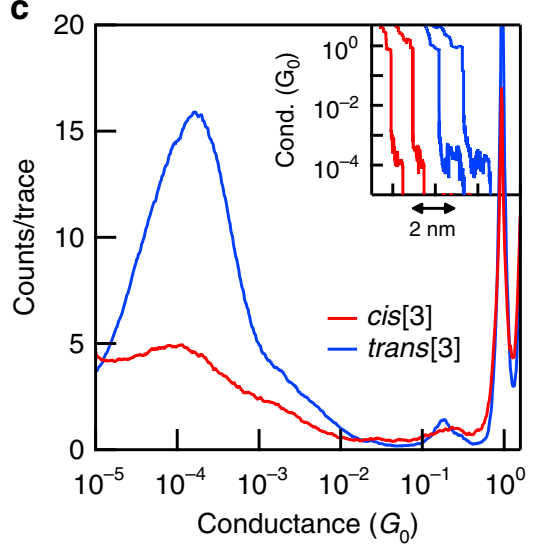

d

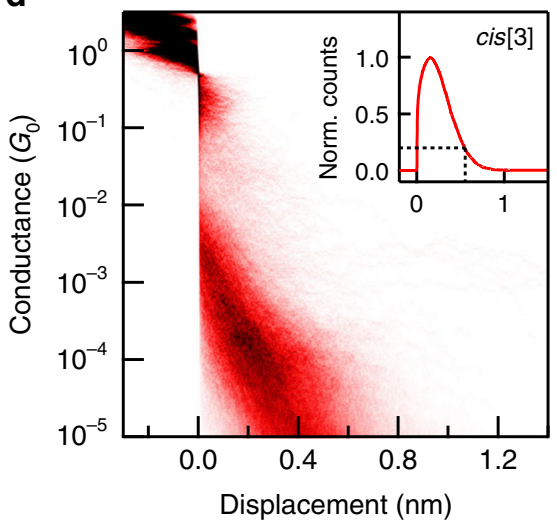

Fig. 1 Conductance measurements of cumulene isomers in solution. a Schematic of the STM setup and the electric field distribution between the STM tip and substrate (green line). b Illustration of the single-molecule junctions with DFT-optimized structures of cis[3] and trans[3] showing distinct S-S distance. The electrodes are model structures not included in the calculations. c Logarithm-binned 1D histograms for cis[3] (red) and trans[3] (blue) measured in tetradecane at a $0.1 \mathrm{~V}$ bias created by compiling 5000 and 9000 conductance traces, respectively. Inset: example conductance vs. displacement traces of cis[3] (red) and trans[3] (blue). d, e 2D conductance-displacement histograms for cis[3] and trans[3], respectively, created by overlaying conductance traces after aligning them at a conductance of $0.5 \mathrm{G}_{0}$. Inset of $\mathbf{d}$ and $\mathbf{e}$ : normalized displacement profiles of cis[3] (red) and trans [3] (blue), respectively, determined from the 2D histograms. The 80th percentile junction length is $0.55 \mathrm{~nm}$ for cis[3] and $1.15 \mathrm{~nm}$ for trans[3] as indicated by the dashed lines

trans[3] solutions, we determine that the $32 \mathrm{~h}$ measurement yields a solution that is $>90 \%$ trans[3] (see Supplementary Fig. 8).

To exclude alternate explanations, we note first that all the measurements are performed in the dark; this rules out photoisomerization. Indeed, measurements in the presence of white light yield $\mathrm{a} \sim$ 40:60 mixture of cis:trans, as determined by $1 \mathrm{H}-\mathrm{NMR}$ and STM-BJ characterizations shown in Supplementary Figs. 9 and 10. Using a combination of variable-temperature $1 \mathrm{H}$ NMR and UV-vis spectroscopy, we find that a thermal equilibrium between cis and trans is achieved at $410 \mathrm{~K}$; this implies a barrier to isomerization likely larger than $1 \mathrm{eV}$ (Supplementary Figs. 11 and 12) 21,22. Importantly, at room temperature, no isomerization is seen in over $72 \mathrm{~h}$ (Supplementary Fig. 2). We also examined each of the two isomers in solution with gold nanoparticles using variable-temperature $1 \mathrm{H}-\mathrm{NMR}$ characterizations (Supplementary Figs. 13 and 14) and we found that the nanoparticles do not affect the dynamics of the isomerization; thus, we exclude the possibility of the isomerization being simply gold-catalyzed. We deduce that the reaction is catalyzed during the STM-BJ process.

The mixed-solution STM results (Fig. 2c) show clearly that we convert not just the fraction of molecules that form molecular junctions but close to the entire solution used in the STM measurements. As we repeat the break-junction measurement 155,000 times over the $32 \mathrm{~h}$ period with a solution that has $\sim 10^{15}$ molecules, the isomerization reaction cannot be occurring only to the small fraction of molecules that are held between the STM tip and substrate. This rules out mechanisms such as those induced by electron tunneling excitations, resonant transport (see Supplementary Fig. 15), or mechanical forces in the junction ${ }^{23-27}$. Finally, we repeat the STM-BJ measurement for a larger quantity of the cis[3] solution for $\sim 240 \mathrm{~h}$, extract this solution from the STM setup, and analyze it using HPLC. We find that the cis:trans ratio for this solution is 30:70 compared with a ratio of 92:8 for a control solution left out for the same amount of time under environmental light (Supplementary Fig. 16). Significantly, the factor of $\sim 27$ increase in the relative ratios demonstrates conclusively that the solution extracted from the STM setup is converted selectively from pure cis to predominantly trans.

To illustrate the generality of these results, we turn to an additional control experiment performed using the same [3] cumulene backbone but without any SMe linkers. The molecules, cis[3]-H and trans[3]-H (Fig. 3a), do not form molecular junctions, as they have no gold-binding linkers. We synthesize these molecules as detailed in Supplementary Methods and carry out STM-BJ measurements (repeatedly forming and breaking 

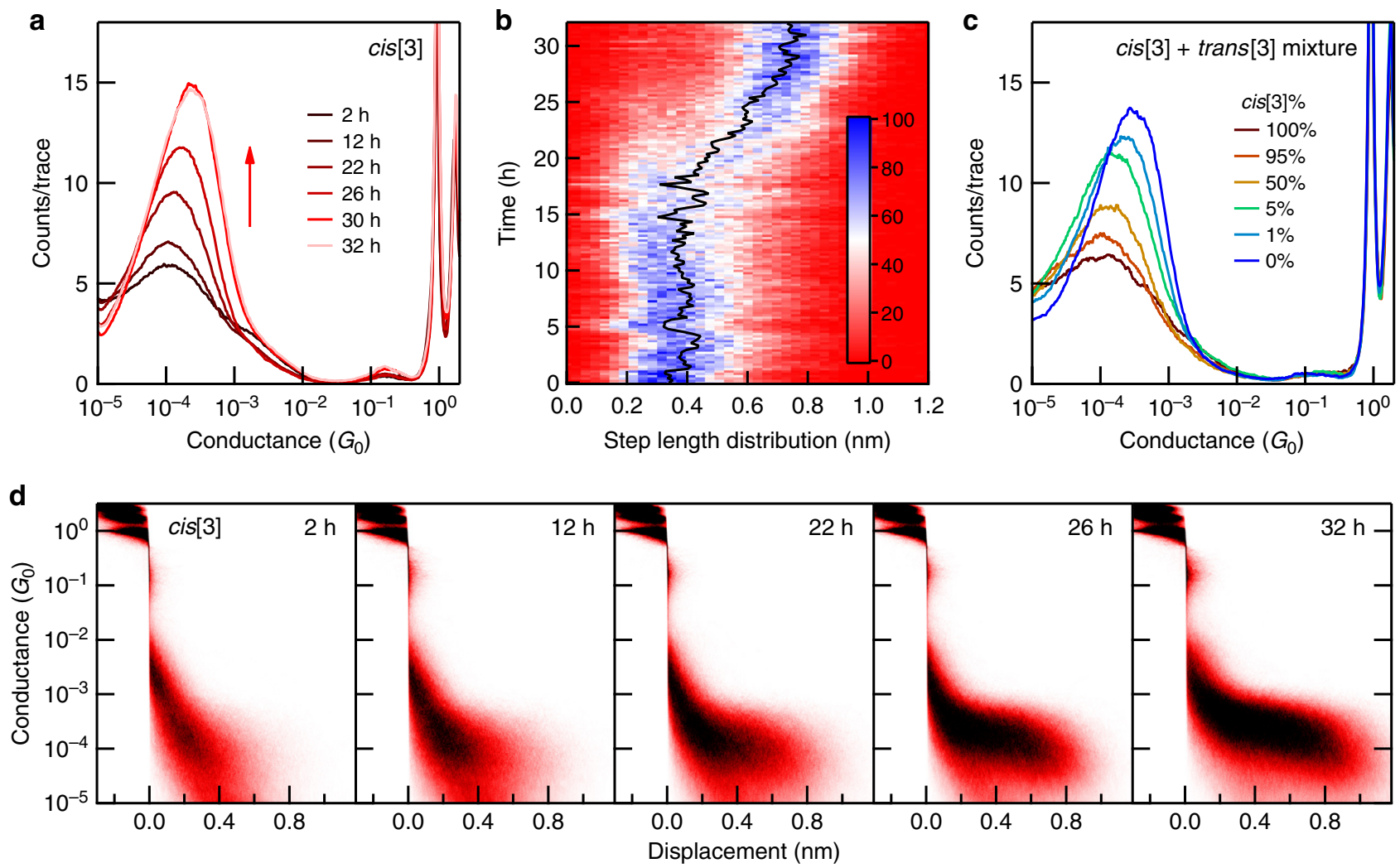

Fig. 2 Isomerization reactions of cumulenes observed during conductance measurements. a Logarithmically binned 1D histograms of 10,000 consecutive conductance traces measured at different times as indicated for cis[3] in tetradecane at a bias of $0.1 \mathrm{~V}$. b Conductance step length distributions as a function of time showing a clear transition from a predominantly shorter plateau at the start of the experiment to longer ones at the end. The image includes step length analysis of 155,000 conductance traces in sets of 1000 traces. The step length is determined by counting data points in each trace over a conductance range between $10^{-3}$ and $10^{-4.9} \mathrm{G}_{0}$. The black line traces the peak position. c Logarithmically binned $1 \mathrm{D}$ histograms for different mixtures of cis[3] and trans[3] in tetradecane measured at a $0.1 \mathrm{~V}$ bias. Each histogram is compiled from 5000 conductance traces. $\mathbf{d} 2 \mathrm{D}$ conductancedisplacement histograms compiled from the same cis[3] data shown in a

gold-point contacts) in the dark with a $0.3 \mathrm{ml}$ solution of cis[3]-H for over $300 \mathrm{~h}$, while applying a $0.5 \mathrm{~V}$ bias. Conductance histograms do not show any clear molecular peaks (Supplementary Fig. 17). However, an HPLC analysis of the solution after the measurement (see Methods) shows that we have created a mixture with a trans[3]-H to cis[3]-H ratio that is roughly 1:1 (Fig. 3b). Comparing this HPLC spectrum with that of the same cis[3]-H solution kept outside the STM-BJ in dark for the same $300 \mathrm{~h}$ demonstrates indisputably that the isomerization reaction is catalyzed during the STM-BJ process. These results further confirm that the isomerization reaction is a solution-phase process where the reactants do not need to form molecular junctions.

STM-BJ measurements involve repeatedly forming and breaking a gold-point contact, while applying a bias voltage. The timeaveraged separation between the tip and substrate is roughly $1 \mathrm{~nm}$; therefore, the solution around the tip/substrate volume is continuously exposed to a high electric field. This field can affect all of the molecules distributed around the electrode gap and over time (owing to the diffusion of the molecules in solution and local turbulence created by the movement of the tip) impact the entire sample. We deduce that the observed isomerization reactions are catalyzed by an electric field. To test whether this is indeed the case, we carry out control experiments with the SMeterminated cis[3] compound. We collected 1000 conductance traces and then either turn off the bias or keep the STM tip retracted by $\sim 1 \mu \mathrm{m}$ from the substrate with $0.1 \mathrm{~V}$ bias applied for a $2 \mathrm{~h}$ period and repeated the cycle for over $36 \mathrm{~h}$. Conductance histograms from these measurements show no conversion from cis[3] to trans[3] (see Supplementary Fig. 18). This therefore confirms that an electric field is necessary to drive the isomerization reaction.

As the electric field distribution depends on the polarity of the solvent, we carry out additional control measurements of cis[3] in different solvents including 1,2,4-trichlorobenzene (TCB, a nonpolar solvent) and propylene carbonate (PC, a polar solvent) with a supporting electrolyte (tetrabutylammonium perchlorate), which screens the field. In TCB, again, we observed a cis-to-trans conversion, which takes $\sim 20 \mathrm{~h}$ (Supplementary Fig. 19). By contrast, there is no conversion in PC even after $50 \mathrm{~h}$ (Supplementary Fig. 20). The control measurement in PC additionally rules out an electrocatalytic mechanism where the reaction is induced by charge transfer at the electrode surface ${ }^{28}$.

Theoretical analysis. To understand how the electric field can catalyze the reaction, we first point out that the cumulenes have many $\pi$-bonds ${ }^{29,30}$. Rather than the standard canonical resonance structure of cis[3] (Fig. 4a), under an applied electric field, the polar zwitterionic resonance structure (Fig. 4b) is enhanced. The important feature to the zwitterionic resonance structure is the (relatively) free rotation around the terminal $\mathrm{C}-\mathrm{C}$ bonds, as the terminal $\pi$-bonds are absent. Thus, an electrostatic polarization, which promotes the zwitterionic resonance structure, can reduce the rotational barrier making isomerization energetically accessible (see Supplementary Note 2 and Supplementary Fig. 21 
a
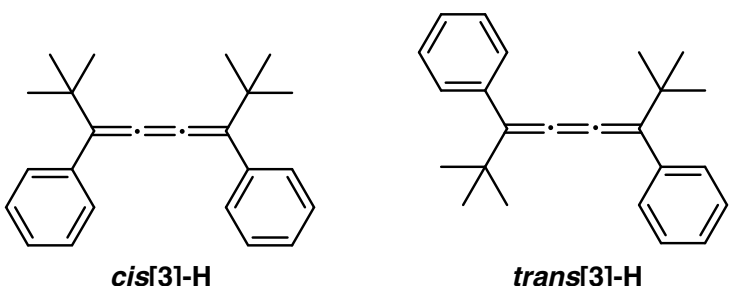

trans[3]-H

b

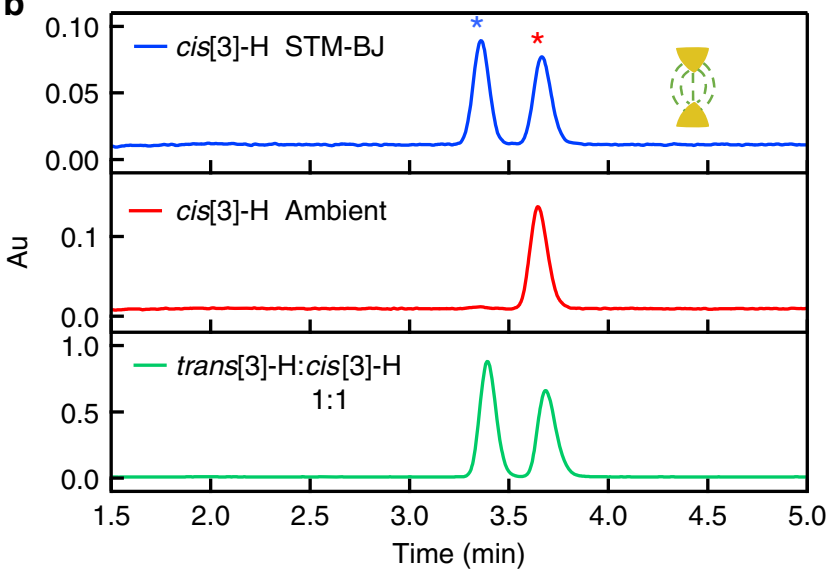

Fig. 3 Isomerization reactions of cumulenes without aurophilic linkers. a Molecular structure of cis[3]-H and trans[3]-H cumulene isomers. b HPLC spectra for cis[3]-H collected after STM-BJ measurements of $\sim 300 \mathrm{~h}$ in TD at $0.5 \mathrm{~V}$ (blue); the same solution kept outside the setup for the same amount of time (red); a 1:1 mixture of cis[3]-H and trans[3]-H (green). The signature peak of cis[3]-H and trans[3]-H is denoted by the red and blue stars, respectively for more discussions). In addition, the applied field dictates the ultimate product distribution, as trans[3] has a larger fieldinduced dipole moment than cis[3] (see Supplementary Fig. 22). Therefore, there is a driving force for the conversion of cis[3] to trans[3] but not the reverse.

To explore this hypothesis more quantitatively, we carry out DFT calculations and explore the energy costs for the isomerization both with and without an electric field. Calculation details are provided in the Methods section and Supplementary Note 2. We find that the barrier for interconversion between cis[3] (dihedral of $0^{\circ}$ ) and trans[3] (dihedral of $180^{\circ}$ ) is $>1 \mathrm{eV}$ (Fig. 4b) in the absence of an electric field. This agrees with the variabletemperature $1 \mathrm{H}$ NMR characterizations presented in Supplementary Figs. 11 and 12. Analyzing the structure (see Supplementary Note 2 for details), we find that the transition state $\left(90^{\circ}\right.$ dihedral angle) has a larger dipole moment than either the cis or the trans form (see Supplementary Fig. 22). This electronic rearrangement in going from either the cis or trans form to the $90^{\circ}$ twisted form accounts, in part, for the large activation energy required for the interconversion. We note that the cis and trans forms are isoenergetic in the absence of a field.

With an electric field of $5 \mathrm{~V} / \mathrm{nm}$ applied along the cumulene backbone, we find that the dipole moment increases in both the cis and trans form as the field stabilizes a charge-separated resonant form (see Supplementary Fig. 22). This electronic reorganization facilitates the isomerization, as the more ionic transition state $\left(90^{\circ}\right.$ dihedral) is stabilized more than the cis[3] reactant by the field. Furthermore, these calculations show that trans[3], which has a much larger dipole moment than cis[3] (28.8 D vs. $19.3 \mathrm{D})$, has a lower energy in a field. This can be attributed to a more efficient charge delocalization over the longer length of the trans isomer compared with the cis. These effects result in a lower activation barrier for isomerization, which favors a

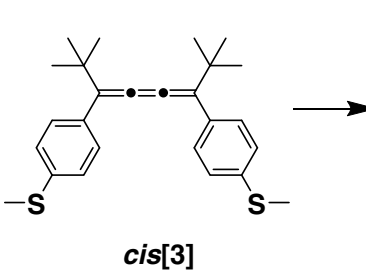

b

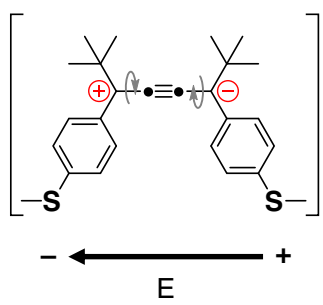

C

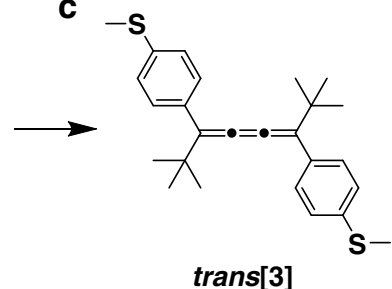

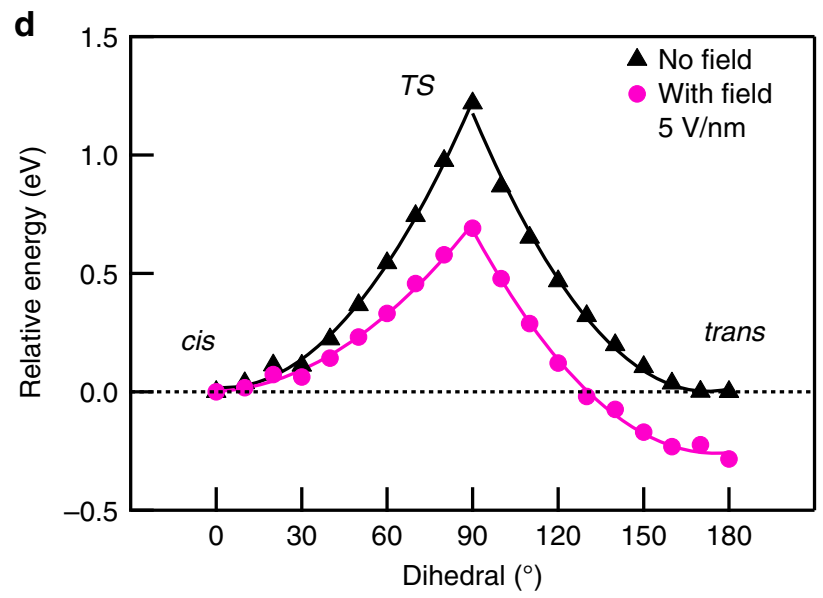

Fig. 4 Calculated reaction energy with and without field. a Standard canonical resonance structure and $\mathbf{b}$ zwitterionic resonance structure of cis[3]. c Standard canonical resonance structure of trans[3]. Gray arrows illustrate the rotation along the C-C bonds. $\mathbf{d}$ Relative energy calculated without (black) and with an electric field of $5 \mathrm{~V} / \mathrm{nm}$ (magenta). Note that the curves are offset vertically to set the energy of cis to 0 
the trans product in an electric field (Fig. 4d), while also impeding the reverse reaction to cis.

To better evaluate the catalytic strength induced by external field, we calculate the relative cis-to-trans reaction rate under different field strengths. As shown in Supplementary Fig. 23, a field of $1 \mathrm{~V} / \mathrm{nm}$ increase the cis-to-trans reaction rate by a factor of 6 . When the field strength is increased to $5 \mathrm{~V} / \mathrm{nm}$, the reaction is accelerated by a factor of 109. This suggests that the electric field is a powerful catalyst for speeding up isomerization. Moreover, we compare these field-induced dipole moments with the permanent dipole of a series of analogous molecules with donor and acceptor substitutions on the two ends. The polarization induced by external field is comparable to that of the molecule with charged functional groups (see Supplementary Fig. 24). The transformation from electrostatic strength to chemical strength provides insights for designing molecular electrostatic catalytic reactions by employing internal field created by chemically engineering molecular polarization.

We note that the fields used in the calculations are considerably larger than what would be expected considering a simple parallel plate capacitor with a separation of $\sim 1 \mathrm{~nm}$ under a $0.1 \mathrm{~V}$ bias for the tip-substrate pair. However, in STM-BJ measurements, the tip is repeatedly brought in and out of contact with the substrate at room temperature. Because of the electrode movements and gold atom reorganization, the distance between electrodes and therefore the field strength is changing during the measurements. The exact electric field around the apex of an atomically sharp STM tip is likely to be larger.

\section{Discussion}

This study demonstrates unambiguously that electric field catalysis can be achieved in a solution environment using STM techniques where large catalytic electric fields can be created between the STM tip and substrate, while also enabling the detection of the reactants/products in situ through singlemolecule conductance measurements. We show that an external electric field can increase the isomerization reaction rate and while also altering its selectivity. Through DFT calculations, we show that the reaction kinetics and thermodynamics are altered by the electrostatic field. The isomerization reaction of the cumulenes that have no aurophilic linkers further demonstrates that the external applied field aligns molecules along the reaction axis, facilitating scalable applications of solution-based electric field-driven catalysis. Our experiments however do not allow us to determine, quantitatively, field-dependent reaction rates, as we cannot determine the magnitude of the field between the STM tip and substrate in the solution environment. Moreover, in contrast with conventional chemical synthesis (which are typically characterized via HPLC or NMR techniques), nanoscale techniques cannot be applied for large-scale catalysis.

\section{Methods \\ STM-BJ measurements. We repeatedly form and break point contact between a gold STM tip and substrate in a dilute solution $(0.1 \mathrm{mM})$ of the target molecule under ambient conditions at room temperature in a custom setup ${ }^{31}$. After the $\mathrm{Au}$ - point contact is broken, a molecule bridges the gap between the gold electrodes to form a single-molecule junction. Conductance is measured as a function of the tip- substrate displacement under an applied bias voltage, yielding traces that reveal molecular-dependent conductance plateaus. With these measurements, we obtain molecular junction-specific conductance by compiling measured traces into 1D conductance histograms and the molecular backbone length from $2 \mathrm{D}$ conductance- displacement histograms.}

HPLC measurements. All samples were resolved by an Agilent 1200 Series ana lytical HPLC equipped with a diode array detector $(220-900 \mathrm{~nm})$ on a CHIRALPAK IA-3 column $(4.6 \mathrm{~mm}$ ID $\times 250 \mathrm{~mm}, 3 \mu \mathrm{m})$ from Chiral Technologies, premixed $0.5 \% \mathrm{CH}_{2} \mathrm{Cl}_{2} /$ hexanes as the mobile phase, $1.00 \mathrm{~mL} / \mathrm{min}$ flow rate, detection $\lambda=350 \mathrm{~nm}$.
DFT calculations. The geometry optimization and calculation of molecular total energy was carried out using the Fritz Haber Institute ab initio molecular simulation (FHI-aims) package ${ }^{32,33}$, using DFT with generalized gradient approximations for exchange-correlations energy developed by Becke, 3-parameter, LeeYang-Parr. The calculations are performed without and with an electric field by determining the energy for optimized structures at a range of dihedral angles.

\section{Data availability}

The data that support the findings of this study not included in the supplementary information document are available from the corresponding author upon reasonable request.

\section{Code availability}

The data that support the findings was acquired using a custom instrument controlled by custom software (Igor Pro, Wavemetrics). The software is available from the corresponding author upon reasonable request.

Received: 21 July 2019; Accepted: 5 September 2019; Published online: 02 October 2019

\section{References}

1. Shaik, S., Mandal, D. \& Ramanan, R. Oriented electric fields as future smart reagents in chemistry. Nat. Chem. 8, 1091-1098 (2016).

2. Aragones, A. C. et al. Electrostatic catalysis of a Diels-Alder reaction. Nature 531, 88-91 (2016)

3. Fried, S. D., Bagchi, S. \& Boxer, S. G. Extreme electric fields power catalysis in the active site of ketosteroid isomerase. Science 346, 1510-1514 (2014).

4. Welborn, V. V., Ruiz Pestana, L. \& Head-Gordon, T. Computational optimization of electric fields for better catalysis design. Nat. Catal. 1, 649-655 (2018).

5. Che, F. et al. Elucidating the roles of electric fields in catalysis: a perspective. ACS Catal. 8, 5153-5174 (2018).

6. Ciampi, S., Darwish, N., Aitken, H. M., Díez-Pérez, I. \& Coote, M. L. Harnessing electrostatic catalysis in single molecule, electrochemical and chemical systems: a rapidly growing experimental tool box. Chem. Soc. Rev. 47, 5146-5164 (2018).

7. Meir, R., Chen, H., Lai, W. \& Shaik, S. Oriented electric fields accelerate DielsAlder reactions and control the endo/exo selectivity. Chem. Phys. Chem. 11, 301-310 (2010).

8. Foroutan-Nejad, C. \& Marek, R. Potential energy surface and binding energy in the presence of an external electric field: modulation of anion- $\pi$ interactions for graphene-based receptors. Phys. Chem. Chem. Phys. 16, 2508-2514 (2014).

9. Lau, V. M., Pfalzgraff, W. C., Markland, T. E. \& Kanan, M. W. Electrostatic control of regioselectivity in $\mathrm{Au}(\mathrm{I})$-catalyzed hydroarylation. J. Am. Chem. Soc. 139, 4035-4041 (2017).

10. Gorin, C. F., Beh, E. S. \& Kanan, M. W. An electric field-induced change in the selectivity of a metal oxide-catalyzed epoxide rearrangement. J. Am. Chem. Soc. 134, 186-189 (2012).

11. Gorin, C. F., Beh, E. S., Bui, Q. M., Dick, G. R. \& Kanan, M. W. Interfacial electric field effects on a carbene reaction catalyzed by Rh porphyrins. J. Am. Chem. Soc. 135, 11257-11265 (2013).

12. Shi, M. W. et al. Measurement of electric fields experienced by urea guest molecules in the 18-crown-6/urea (1:5) host-guest complex: an experimental reference point for electric-field-assisted catalysis. J. Am. Chem. Soc. 141, 3965-3976 (2019).

13. Meyers, F., Marder, S. R., Pierce, B. M. \& Bredas, J. L. Electric field modulated nonlinear optical properties of donor-acceptor polyenes: sum-over-states investigation of the relationship between molecular polarizabilities (.alpha., .beta., and .gamma.) and bond length alternation. J. Am. Chem. Soc. 116, 10703-10714 (1994).

14. Shaik, S., de Visser, S. P. \& Kumar, D. External electric field will control the selectivity of enzymatic-like bond activations. J. Am. Chem. Soc. 126, 11746-11749 (2004).

15. Alemani, M. et al. Electric field-induced isomerization of azobenzene by STM. J. Am. Chem. Soc. 128, 14446-14447 (2006).

16. Wendinger, D. \& Tykwinski, R. R. Odd [n]cumulenes $(\mathrm{n}=3,5,7,9)$ : synthesis, characterization, and reactivity. Acc. Chem. Res. 50, 1468-1479 (2017).

17. Xu, B. Q. \& Tao, N. J. Measurement of single-molecule resistance by repeated formation of molecular junctions. Science 301, 1221-1223 (2003).

18. Venkataraman, L. et al. Single-molecule circuits with well-defined molecular conductance. Nano Lett. 6, 458-462 (2006).

19. Januszewski, J. A. \& Tykwinski, R. R. Synthesis and properties of long [n] cumulenes ( $\mathrm{n} \geq 5$ ). Chem. Soc. Rev. 43, 3184-3203 (2014). 
20. Kamenetska, M. et al. Formation and evolution of single-molecule junctions. Phys. Rev. Lett. 102, 126803 (2009).

21. Bühringer, M. U. et al. Double bonds? Studies on the barrier to rotation about the cumulenic $\mathrm{C}=\mathrm{C}$ bonds of tetraaryl[$[\mathrm{n}]$ cumulenes $(\mathrm{n}=3,5,7,9)$. Angew. Chem. Int. Ed. 57, 8321-8325 (2018).

22. Jarowski, P. D., Diederich, F. \& Houk, K. N. Butatrienes as extended alkenes: barriers to internal rotation and substitution effects on the stabilities of the ground states and transition states. J. Phys. Chem. A 110, 7237-7246 (2006).

23. Henzl, J., Mehlhorn, M., Gawronski, H., Rieder, K. H. \& Morgenstern, K. Reversible cis-trans isomerization of a single azobenzene molecule. Angew. Chem. Int. Ed. 45, 603-606 (2006).

24. Liljeroth, P., Repp, J. \& Meyer, G. Current-induced hydrogen tautomerization and conductance switching of naphthalocyanine molecules. Science 317, 1203-1206 (2007).

25. Choi, B. Y. et al. Conformational molecular switch of the azobenzene molecule: a scanning tunneling microscopy study. Phys. Rev. Lett. 96, 156106 (2006).

26. Zang, Y. et al. Resonant transport in single diketopyrrolopyrrole junctions. J. Am. Chem. Soc. 140, 13167-13170 (2018).

27. Turanský, R., Konôpka, M., Doltsinis, N. L., Štich, I. \& Marx, D. Switching of functionalized azobenzene suspended between gold tips by mechanochemical, photochemical, and opto-mechanical means. Phys. Chem. Chem. Phys. 12, 13922-13932 (2010).

28. Goulet-Hanssens, A. et al. Electrocatalytic $Z \rightarrow E$ isomerization of azobenzenes. J. Am. Chem. Soc. 139, 335-341 (2017).

29. Auffrant, A., Jaun, B., Jarowski, P. D., Houk, K. N. \& Diederich, F. Peralkynylated buta-1,2,3-trienes: exceptionally low rotational barriers of cumulenic $\mathrm{C}=\mathrm{C}$ bonds in the range of those of peptide $\mathrm{C}-\mathrm{N}$ bonds. Chem Eur. J. 10, 2906-2911 (2004).

30. Gawel, P. et al. Push-pull buta-1,2,3-trienes: exceptionally low rotational barriers of cumulenic $\mathrm{C}=\mathrm{C}$ bonds and proacetylenic reactivity. Chem. Eur. J. 21, 6215-6225 (2015).

31. Venkataraman, L., Klare, J. E., Nuckolls, C., Hybertsen, M. S. \& Steigerwald, M. L. Dependence of single-molecule junction conductance on molecular conformation. Nature 442, 904-907 (2006)

32. Havu, V., Blum, V., Havu, P. \& Scheffler, M. Efficient integration for allelectron electronic structure calculation using numeric basis functions. J. Comp. Phys. 228, 8367-8379 (2009).

33. Blum, V. et al. Ab initio molecular simulations with numeric atom-centered orbitals. Comput. Phys. Commun. 180, 2175-2196 (2009).

\section{Acknowledgements}

We thank Marc H. Garner for discussions. This research was supported primarily by the National Science Foundation grant CHE-1764256 and DMR-1807580. This research was supported in part by the Center for Precision Assembly of Superstratic and Superatomic Solids at Columbia University, an NSF MRSEC (award number DMR-1420634). The synthesis and characterization of the molecules in this work was supported by the Department of Energy grant DE-SC0019440. Q.Z. and H.L. acknowledge financial support from Shanghai University of Electric Power, the Science and Technology Commission of Shanghai Municipality (14DZ2261000).

\section{Author contributions}

Y.Z., Q.Z., M.L.S., C.N., and L.V. conceived the study. Y.Z., T.F., and L.V. carried out the STM measurements, data analysis, and DFT calculations. Q.Z., B.F., J.Y., and F.N. synthesized and characterized the compounds and reaction products. L.V., C.N., M.L.S., and H.L. supervised the research. The manuscript was written by Y.Z. and L.V. with contributions from all other authors.

\section{Competing interests}

The authors declare no competing interests.

\section{Additional information}

Supplementary information is available for this paper at https://doi.org/10.1038/s41467019-12487-w.

Correspondence and requests for materials should be addressed to Q.Z., M.L.S., C.N. or L.V.

Peer review information Nature Communications thanks the anonymous reviewer(s) for their contribution to the peer review of this work.

Reprints and permission information is available at http://www.nature.com/reprints

Publisher's note Springer Nature remains neutral with regard to jurisdictional claims in published maps and institutional affiliations.

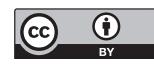

Open Access This article is licensed under a Creative Commons Attribution 4.0 International License, which permits use, sharing, adaptation, distribution and reproduction in any medium or format, as long as you give appropriate credit to the original author(s) and the source, provide a link to the Creative Commons license, and indicate if changes were made. The images or other third party material in this article are included in the article's Creative Commons license, unless indicated otherwise in a credit line to the material. If material is not included in the article's Creative Commons license and your intended use is not permitted by statutory regulation or exceeds the permitted use, you will need to obtain permission directly from the copyright holder. To view a copy of this license, visit http://creativecommons.org/ licenses/by/4.0/

(c) The Author(s) 2019 